Editorial

\title{
Acknowledgement to Reviewers of OBM Geriatrics in 2021
}

OBM Geriatrics Editorial Office

LIDSEN Publishing Inc., 2000 Auburn Drive, One Chagrin Highlands, Suite 200, Beachwood, OH, USA;

E-Mail: geriatrics@lidsen.com

OBM Geriatrics

2022, volume 6, issue 1

doi:10.21926/obm.geriatr.2201186
Received: January 06, 2022

Accepted: January 06, 2022

Published: January 06, 2022

\begin{abstract}
The editors of $O B M$ Geriatrics would like to express their sincere gratitude to the following reviewers for assessing manuscripts in 2021 . We greatly appreciate the contribution of expert reviewers, which is crucial to the journal's editorial process. We aim to recognize reviewer contributions through several mechanisms, of which the annual publication of reviewer names is one. Reviewers receive a voucher entitling them to a discount on their next LIDSEN publication and can download a certificate of recognition directly from our submission system. Additionally, reviewers can sign up to the service Publons (https://publons.com) to receive recognition. Of course, in these initiatives we are careful not to compromise reviewer confidentiality. Many reviewers see their work as a voluntary and often unseen part of their role as researchers. We are grateful to the time reviewers donate to our journals and the contribution they make.
\end{abstract}

If you are interested in becoming a reviewer for OBM Geriatrics, see the link at the bottom of the webpage http://www.lidsen.com/joinus.

The following reviewed for OBM Geriatrics in 2021:

\begin{tabular}{|l|l|l|}
\hline Alfaro, Ana & Gutschow, C. A. & Ogawa, Elisa \\
\hline Allen, Jacqui & Hagberg, Gisela E. & Ogliari, Giulia \\
\hline Amore, Mario & Harvey, Idethia S & Patil, Shankargouda \\
\hline Barnes, Ryan P. & Hayslip, Bert & Peres, Alessandra \\
\hline Bertolucci, Paulo Henrique Ferre & Hlavka, Jakub & Perriot, Sylvain \\
\hline
\end{tabular}

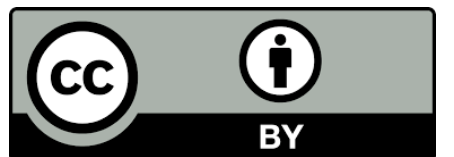

(C) 2022 by the author. This is an open access article distributed under the conditions of the Creative Commons by Attribution License, which permits unrestricted use, distribution, and reproduction in any medium or format, provided the original work is correctly cited. 


\begin{tabular}{|l|l|l|}
\hline Boaden, Elizabeth & Holsinger, R. M. Damian & Petretto, DonatellaR. \\
\hline Bollig, Georg & Huillard, Olivier & Radler, DianeRigassio \\
\hline Boniewska-Bernacka, Ewa & Hurtado, Olivia & Reddy, hemachandra \\
\hline Bonner, Julie & Ikai-Tani, Saeko & Rizza, Stefano \\
\hline Brenner, Steven R. & Jardine, Marie & Robinson, Jackie \\
\hline Breslin, Monique & Jockwitz, Christiane & Roxanas, MiltonG. \\
\hline Brooks, Deborah & Kemp, Michael G. & Russo, Andrea \\
\hline Byles, Julie E. & Kirshner, Howard S. & Ruzieh, Mohammed \\
\hline Cakir, Aysen & Koreli, Alexandra & Saleh, Christian \\
\hline Carter, Ben & Langdahl, Bente & Saretzki, Gabriele \\
\hline Codd, Veryan & Laver, Kate & Sfeir, Jad \\
\hline De Paepe, Boel & Legrand, Érick & Shang, Yuan \\
\hline DeCaporale-Ryan, Lauren N. & Lilleker, James B. & Shen, Xiaoli \\
\hline Dening, Karen Harrison & Littleford, Angela & Shi, Jing \\
\hline Di Raimondo, Domenico & Lundervold, Duane & Simonetti, Antonella F. \\
\hline Duncan, Robin & Mammi, Patrizia & Smithard, David G. \\
\hline Ebihara, Takae & Mañas-Martínez, Ana B. & Tao, Jungz \\
\hline Economou, Alexandra & Mao, Xiaoyuan & Terzoni, Stefano \\
\hline Fionda, Bruno & Middeldorp, Melissa E. & Tham, Tony C. K. \\
\hline Fouquerel, Elise & Monji, Akira & Tohara, Haruka \\
\hline Fu, Dehao & Montorio, Ignacio & Van Puymbroeck, Marieke \\
\hline Gadbail, Amol R. & Moonaz, Steffany & Viaña, John Noel M. \\
\hline Galik, Elizabeth M. & Muszalik, Marta & Westmark, Signe \\
\hline Galli, Francesco & Nadimi, Navid & Whitney, Daniel G. \\
\hline Garcia-Ibarbia, Carmen & Nakabeppu, Yusaku & Zhang, Jingzhe \\
\hline Gazdag, Gábor & Nogués, Xavier & Zibelman, Matthew R. \\
\hline Gibb, Robbin & Nyffeler, Thomas & Zou, Kaiwen \\
\hline Greenwald, Bruce D. & & \\
\hline
\end{tabular}

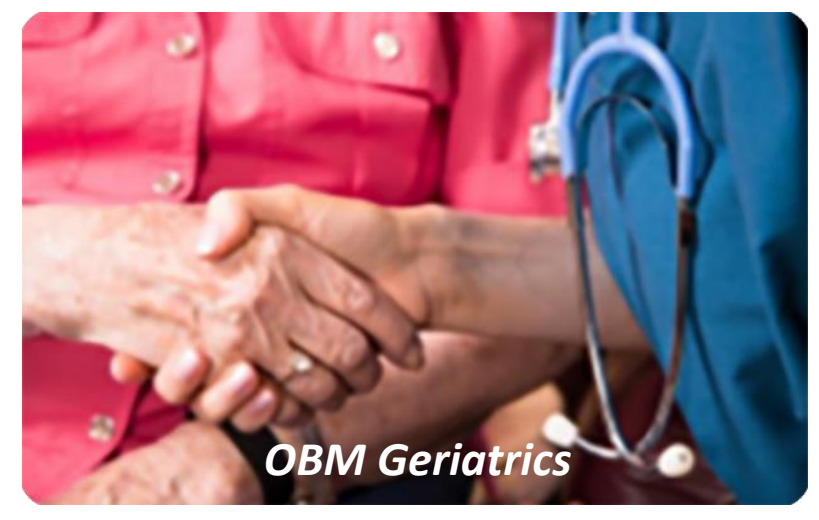

Enjoy $O B M$ Geriatrics by:

1. Submitting a manuscript

2. Joining in volunteer reviewer bank

3. Joining Editorial Board

4. Guest editing a special issue

For more details, please visit:

http://www.lidsen.com/journals/geriatrics 